\title{
Substituent Effects Control the Self-Association of Molecular Clips in the Crystalline State
}

\author{
by Zhi-Guo Wang ${ }^{\dagger}$, Bao-Han Zhou ${ }^{\dagger}$,Yun-Feng Chen ${ }^{\dagger}$, Guo-Dong Yin ${ }^{\dagger}$, Yi-Tao Li $^{\dagger}$, \\ An-Xin $W^{\dagger, *}$, and Lyle Isaacs ${ }^{* * *}$
}

Table of Contents

Pages

General Experimental S2

Synthetic Procedures and Characterization

$\mathrm{S} 2-\mathrm{S} 4$

References

S4

Selected ${ }^{1} \mathrm{H}$ and ${ }^{13} \mathrm{C}$ NMR spectra

$\mathrm{S} 5-\mathrm{S} 22$ 
General Experimental. General experimental details have been published previously. ${ }^{1,2}$ Compounds $1,{ }^{3} \mathbf{2 b},{ }^{4} \mathbf{2 c},{ }^{5} \mathbf{2 d},{ }^{6,7} \mathbf{2 e}, \mathbf{2 f},{ }^{8} \mathbf{5 a},{ }^{9} \mathbf{5 b},{ }^{9}$ and $\mathbf{5} \mathbf{c}^{2}$ were prepared according to the literature procedures. Compound $\mathbf{2 a}$ is commercially available.

\section{Synthetic Procedures and Characterization.}

Compound 2e. A mixture of 4-nitro-o-xylene (15.1 g, $100 \mathrm{mmol})$ and N-bromosuccinimide (35.6 g, $200 \mathrm{mmol})$ in 1,2-dichloroethane $(200 \mathrm{~mL})$ was refluxed under illumination $(250 \mathrm{~W}$ incandescent bulb) for $1 \mathrm{~h}$. The reaction mixture was washed with water $(2 \times 200 \mathrm{~mL})$, dried over anh. $\mathrm{MgSO}_{4}$, and concentrated. Flash chromatography $\left(\mathrm{SiO}_{2}, \mathrm{Hexane} / \mathrm{EtOAc} 30: 1\right)$ gave crude 1,2-bis(bromomethyl)-5-nitrobenzene. Recrystallization from EtOH gave pure product $(14.5 \mathrm{~g}, 47 \%)$ as a white solid. M.p. $79-80{ }^{\circ} \mathrm{C}$ (from EtOH). TLC (Hexane/EtOAc 10:1) $R_{\mathrm{f}}$ 0.37. IR (KBr, cm $\left.{ }^{-1}\right): 3095 \mathrm{w}, 3048 \mathrm{w}, 1610 \mathrm{~m}, 1588 \mathrm{~m}, 1524 \mathrm{~s}, 1485 \mathrm{~m}, 1349 \mathrm{~s}, 1318 \mathrm{~m}, 1216 \mathrm{~m}$, $1184 \mathrm{~m} .{ }^{1} \mathrm{H}$ NMR $\left(400 \mathrm{MHz}, \mathrm{CDCl}_{3}\right): 8.23(\mathrm{~s}, 1 \mathrm{H}), 8.13(\mathrm{~d}, J=8.4,1 \mathrm{H}), 7.54(\mathrm{~d}, J=8.4$, $1 \mathrm{H}), 4.65$ (s, 2H), $4.64(\mathrm{~s}, 2 \mathrm{H}) .{ }^{13} \mathrm{C} \mathrm{NMR}\left(100 \mathrm{MHz}, \mathrm{CDCl}_{3}\right)$ : 148.4, 143.8, 138.7, 132.5, 126.3, 124.5, 28.4, 27.9. MS (EI): $m / z 309\left(15,[\mathrm{M}]^{+}\right), 228$ (100). HR-MS (EI): $\mathrm{m} / \mathrm{z}$ $306.8847\left([\mathrm{M}]^{+}, \mathrm{C}_{8} \mathrm{H}_{7}^{79} \mathrm{Br}_{2} \mathrm{NO}_{2}\right.$, calcd 306.8844).

Compound 3a. ${ }^{10}$ Compound 1 (1.43 g, $\left.5.00 \mathrm{mmol}\right)$ was dissolved in anh. DMSO (30 mL) under $\mathrm{N}_{2}$ and $t$-BuOK $(2.24 \mathrm{~g}, 20.0 \mathrm{mmol})$ was added in one portion. After stirring for 15 min., 2a $(1.59 \mathrm{~g}, 11.0 \mathrm{mmol})$ was added in one portion and stirring was continued for $6 \mathrm{~h}$. The reaction mixture was poured into $0.1 \mathrm{~N} \mathrm{HCl}(500 \mathrm{~mL})$ and extracted with EtOAc $(3 \times$ $400 \mathrm{~mL})$. The extracts were washed with brine $(2 \times 300 \mathrm{~mL})$ and dried over anh. $\mathrm{MgSO}_{4}$. After filtration and rotary evaporation the residue was purified by flash chromatography $\left(\mathrm{SiO}_{2}, \mathrm{CHCl}_{3} / \mathrm{MeOH}, 100: 1\right)$ to give 3a $(0.860 \mathrm{~g}, 1.75 \mathrm{mmol}, 35 \%)$. M.p. $271-272{ }^{\circ} \mathrm{C} .{ }^{1} \mathrm{H}$ NMR (400 MHz, $\left.\mathrm{CDCl}_{3}\right): 7.30-7.10(\mathrm{~m}, 8 \mathrm{H}), 4.76(\mathrm{~d}, J=16.0,4 \mathrm{H}), 4.42(\mathrm{~d}, J=16.0,4 \mathrm{H})$, $4.28(\mathrm{q}, J=7.2,4 \mathrm{H}), 1.33(\mathrm{t}, J=7.2,6 \mathrm{H})$.

Compound 3c. Compound 1 (1.43 g, $5.00 \mathrm{mmol})$ was dissolved in anh. DMSO $(30 \mathrm{~mL})$ under $\mathrm{N}_{2}$ and $t$-BuOK $(2.24 \mathrm{~g}, 20.0 \mathrm{mmol})$ was added. After stirring for $15 \mathrm{~min}$., $2 \mathrm{c}(2.23 \mathrm{~g}$, $11.00 \mathrm{mmol})$ was added in one portion and stirring was continued for $6 \mathrm{~h}$. The reaction mixture was poured into $0.1 \mathrm{~N} \mathrm{HCl}(500 \mathrm{~mL})$ and extracted with EtOAc $(3 \times 400 \mathrm{~mL})$. The extracts were washed with brine $(2 \times 300 \mathrm{~mL})$ and dried over anh. $\mathrm{MgSO}_{4}$. After filtration and rotary evaporation the residue was purified by flash chromatography $\left(\mathrm{SiO}_{2}\right.$, $\left.\mathrm{CHCl}_{3} / \mathrm{MeOH}, 100: 1\right)$ to give $3 \mathrm{c}(1.09 \mathrm{~g}, 2.00 \mathrm{mmol}, 40 \%)$. M.p. $>300{ }^{\circ} \mathrm{C}$. TLC $\left(\mathrm{CHCl}_{3}\right) R_{\mathrm{f}}$ 0.20. IR (KBr, cm $\left.{ }^{-1}\right): 3415 \mathrm{w}, 2977 \mathrm{w}, 1757 \mathrm{~s}, 1724 \mathrm{~s}, 1711 \mathrm{~s}, 1462 \mathrm{~s}, 1261 \mathrm{~s}, 1021 \mathrm{~m}$. ${ }^{1} \mathrm{H}$ NMR $\left(400 \mathrm{MHz}, \mathrm{DMSO}-\mathrm{d}_{6}\right): 6.95(\mathrm{~s}, 4 \mathrm{H}), 4.48(\mathrm{~d}, J=16.0,4 \mathrm{H}), 4.39(\mathrm{~d}, J=16.0,4 \mathrm{H}), 4.26(\mathrm{q}, J$ $=7.2,4 \mathrm{H}), 2.07(\mathrm{~s}, 12 \mathrm{H}), 1.25(\mathrm{t}, J=7.2,6 \mathrm{H}) .{ }^{13} \mathrm{C}$ NMR $\left(100 \mathrm{MHz}, \mathrm{DMSO}-\mathrm{d}_{6}\right): 166.2$, 156.1 $136.3,135.2,131.4,80.9,64.3,45.2,19.6,14.6$. EI-MS: $m / z .547\left(75,[\mathrm{M}+1]^{+}\right), 473$ (100).

Compound 3d. Compound 1 (1.43 g. $5.00 \mathrm{mmol})$ was dissolved in anh. DMSO $(30 \mathrm{~mL})$ under $\mathrm{N}_{2}$ and $t$-BuOK $(2.24 \mathrm{~g}, 20.0 \mathrm{mmol})$ was added. After stirring for $15 \mathrm{~min}$., $2 \mathbf{d}$ (3.56 $\mathrm{g}, 11.0 \mathrm{mmol}$ ) was added in one portion and stirring was continued for $6 \mathrm{~h}$. The reaction mixture was poured into $0.1 \mathrm{~N} \mathrm{HCl}(500 \mathrm{~mL})$ and extracted with EtOAc $(3 \times 400 \mathrm{~mL})$. The extracts were washed with brine $(2 \times 300 \mathrm{~mL})$ and dried over anh. $\mathrm{MgSO}_{4}$. After filtration and rotary evaporation the residue was purified by flash chromatography $\left(\mathrm{SiO}_{2}\right.$, $\left.\mathrm{CHCl}_{3} / \mathrm{MeOH}, 100: 1\right)$ to give 3d $(1.43 \mathrm{~g}, 2.35 \mathrm{mmol}, 47 \%)$. M.p. $295-296{ }^{\circ} \mathrm{C}$. ${ }^{1} \mathrm{H}$ NMR $\left(400 \mathrm{MHz}, \mathrm{CDCl}_{3}\right): 6.72(\mathrm{~s}, 6 \mathrm{H}), 5.26(\mathrm{~d}, J=15.6,4 \mathrm{H}), 4.22(\mathrm{~d}, J=15.6,4 \mathrm{H}), 4.12(\mathrm{q}, J=$ $7.2,4 \mathrm{H}), 3.80(\mathrm{~s}, 6 \mathrm{H}), 1.24(\mathrm{t}, J=7.2,6 \mathrm{H})$. 
Compounds 3eC and $( \pm)-3 e T$. Compound $1(2.86 \mathrm{~g}$. $10.0 \mathrm{mmol})$ was dissolved in anh. DMSO (100 mL) under $\mathrm{N}_{2}$ and $t$-BuOK $(4.48 \mathrm{~g}, 40.0 \mathrm{mmol})$ was added. After stirring for 15 min., $2 \mathrm{e}(6.80 \mathrm{~g}, 22.0 \mathrm{mmol})$ was added in one portion and stirring was continued for $6 \mathrm{~h}$. The reaction mixture was poured into $0.1 \mathrm{~N} \mathrm{HCl}(1 \mathrm{~L})$ and extracted with EtOAc $(3 \times 500 \mathrm{~mL})$. The extracts were washed with brine $(2 \times 400 \mathrm{~mL})$ and dried over anh. $\mathrm{MgSO}_{4}$. After filtration and rotary evaporation the residue was purified by flash chromatographed $\left(\mathrm{SiO}_{2}\right.$, $\left.\mathrm{CHCl}_{3}\right)$ to give $\mathbf{3 e C}(0.75 \mathrm{~g}, 1.30 \mathrm{mmol}, 13 \%)$ and $( \pm)-3 \mathbf{e T}(1.04 \mathrm{~g}, 1.80 \mathrm{mmol}, 18 \%)$. Compound 3eC: M.p. $280-281{ }^{\circ}$ C. TLC $\left(\mathrm{CHCl}_{3} / \mathrm{MeOH}, 100: 1\right) R_{\mathrm{f}} 0.38$. IR $\left(\mathrm{KBr}, \mathrm{cm}^{-1}\right)$ : 2924w, 2852w, 1731s, 1717s, 1649s, 1537m, 1259m, 1020w. ${ }^{1} \mathrm{H}$ NMR (400 MHz, $\mathrm{CDCl}_{3}$ ): $8.10(\mathrm{~s}, 2 \mathrm{H}), 7.99(\mathrm{~d}, J=8.8,2 \mathrm{H}), 7.42(\mathrm{~d}, J=8.8,2 \mathrm{H}), 4.91(\mathrm{~d}, J=16.0,2 \mathrm{H}), 4.89(\mathrm{~d}, J=$ $16.0,2 \mathrm{H}), 4.51(\mathrm{~d}, J=16.0,2 \mathrm{H}), 4.50(\mathrm{~d}, J=16.0,2 \mathrm{H}), 4.37(\mathrm{q}, J=7.2,4 \mathrm{H}), 1.38(\mathrm{t}, J=7.2$, $6 \mathrm{H}) .{ }^{13} \mathrm{C} \mathrm{NMR}\left(100 \mathrm{MHz}, \mathrm{CDCl}_{3}\right): 165.1,155.3,147.3,143.1,138.1,130.7,124.4,123.2$, 79.7, 63.8, 44.8, 14.0, (only 12 of the 13 expected resonances were observed). EI-MS: $\mathrm{m} / \mathrm{z}$ $581\left(23,[\mathrm{M}+1]^{+}\right), 507(100)$. Compound ( \pm -3eT: M.p. $189-191{ }^{\circ} \mathrm{C} . \quad \mathrm{TLC}\left(\mathrm{CHCl}_{3} / \mathrm{MeOH}\right.$, $100: 1) R_{\mathrm{f}} 0.42$. IR (KBr, cm $\left.{ }^{-1}\right): 2924 \mathrm{~m}, 2854 \mathrm{w}, 1752 \mathrm{~s}, 1712 \mathrm{~s}, 1527 \mathrm{~s}, 1456 \mathrm{~s}, 1150 \mathrm{~m}, 1017 \mathrm{~m}$. ${ }^{1} \mathrm{H} \mathrm{NMR}\left(400 \mathrm{MHz}, \mathrm{CDCl}_{3}\right): 8.11(\mathrm{~s}, 2 \mathrm{H}), 8.00(\mathrm{~d}, J=8.0,2 \mathrm{H}), 7.44(\mathrm{~d}, J=8.0,2 \mathrm{H}), 4.90(\mathrm{~d}$, $J=16.0,4 \mathrm{H}), 4.51(\mathrm{~d}, J=16.0,4 \mathrm{H}), 4.37(\mathrm{q}, J=7.2,4 \mathrm{H}), 1.38(\mathrm{t}, J=7.2,6 \mathrm{H}) .{ }^{13} \mathrm{C}$ NMR $\left(100 \mathrm{MHz}, \mathrm{CDCl}_{3}\right): 165.1,155.2,147.2,143.2,138.0,130.7,124.4,123.2,79.6,63.8,44.8$, 14.0. EI-MS: $m / z 581\left(13,[\mathrm{M}+1]^{+}\right), 508(100)$.

Compounds 3fC and 3fT. Compound 1 (2.86 g. $10.0 \mathrm{mmol})$ was dissolved in anh. DMSO $(100 \mathrm{~mL})$ under $\mathrm{N}_{2}$ and $t$-BuOK (4.48 g, $\left.40.0 \mathrm{mmol}\right)$ was added. After stirring for $15 \mathrm{~min} ., 2 \mathbf{2 f}$ $(7.46 \mathrm{~g}, 22.0 \mathrm{mmol})$ was added in one portion and stirring was continued for $6 \mathrm{~h}$. The reaction mixture was poured into $0.1 \mathrm{~N} \mathrm{HCl}(1 \mathrm{~L})$ and extracted with EtOAc $(3 \times 500 \mathrm{~mL})$. The extracts were washed with brine $(2 \times 400 \mathrm{~mL})$ and dried over anh. $\mathrm{MgSO}_{4}$. After filtration and rotary evaporation the residue was purified by flash chromatography $\left(\mathrm{SiO}_{2}, \mathrm{CHCl}_{3}\right)$ to give 3fC $(0.80 \mathrm{~g}, 1.25 \mathrm{mmol}, 13 \%)$ and $( \pm)-3 f T(1.09 \mathrm{~g}, 1.70 \mathrm{mmol}, 17 \%)$. Compound 3fC: M.p. $>300{ }^{\circ} \mathrm{C}$. TLC $\left(\mathrm{CHCl}_{3} / \mathrm{MeOH}, 100: 1\right) R_{\mathrm{f}} 0.21$. IR $\left(\mathrm{KBr}, \mathrm{cm}^{-1}\right): 2980 \mathrm{w}, 2852 \mathrm{w}, 1754 \mathrm{~s}$, $1726 \mathrm{~s}, 1520 \mathrm{~s}, 1458 \mathrm{~s}, 1278 \mathrm{~s}, 1066 \mathrm{~m} .{ }^{1} \mathrm{H}$ NMR (400 MHz, DMSO- $\left.d_{6} / \mathrm{CF}_{3} \mathrm{COOH}\right): 7.80(\mathrm{~d}, J$ $=8.8,2 \mathrm{H}), 7.10(\mathrm{~d}, J=8.8,2 \mathrm{H}), 5.31(\mathrm{~d}, J=16.4,2 \mathrm{H}), 5.18(\mathrm{~d}, J=16.4,2 \mathrm{H}), 4.67(\mathrm{~d}, J=$ $16.4,2 \mathrm{H}), 4.34(\mathrm{~d}, J=16.4,2 \mathrm{H}), 4.21(\mathrm{q}, J=7.2,4 \mathrm{H}), 3.89(\mathrm{~s}, 6 \mathrm{H}), 1.28(\mathrm{t}, J=7.2,6 \mathrm{H}) .{ }^{13} \mathrm{C}$ NMR (100 MHz, $\left.\mathrm{CF}_{3} \mathrm{COOD}\right): 165.8,161.0,159.2,158.3,142.2,133.2,127.4,125.9,110.6$, 81.2, 65.8, 55.3, 38.8, 36.5, 12.1. ${ }^{13} \mathrm{C}$ NMR (400 MHz, $\left.\mathrm{CF}_{3} \mathrm{COOD}\right): 162.3,161.8,161.4$, 160.8, 118.4, 115.6, 112.8, 110.0. EI-MS: $m / z 641\left(89,[\mathrm{M}+\mathrm{H}]^{+}\right), 176(100)$. Compound ( \pm$)-$ 3eT: M.p. $>300{ }^{\circ} \mathrm{C}$. TLC $\left(\mathrm{CHCl}_{3} / \mathrm{MeOH}, 100: 1\right) R_{\mathrm{f}} 0.24$. IR $\left(\mathrm{KBr}, \mathrm{cm}^{-1}\right): 2980 \mathrm{w}, 2857 \mathrm{w}$, $1748 \mathrm{~s}, 1730 \mathrm{~s}, 1521 \mathrm{~s}, 1459 \mathrm{~s}, 1276 \mathrm{~s}, 1065 \mathrm{~s} .{ }^{1} \mathrm{H}$ NMR $\left(400 \mathrm{MHz}, \mathrm{DMSO}-d_{6} / \mathrm{CF}_{3} \mathrm{COOH}\right)$ : $7.78(\mathrm{~d}, J=8.8,2 \mathrm{H}), 7.05(\mathrm{~d}, J=8.8,2 \mathrm{H}), 5.37(\mathrm{~d}, J=16.4,2 \mathrm{H}), 5.29(\mathrm{~d}, J=16.4,2 \mathrm{H}), 4.64$ $(\mathrm{d}, J=16.4,2 \mathrm{H}), 4.35-4.25(\mathrm{~m}, 6 \mathrm{H}), 3.89(\mathrm{~s}, 6 \mathrm{H}), 1.30(\mathrm{t}, J=7.2,6 \mathrm{H}) .{ }^{13} \mathrm{C}$ NMR $(100 \mathrm{MHz}$, $\left.\mathrm{CF}_{3} \mathrm{COOD}\right): 165.8,158.6,142.3,133.1,127.2$, 125.9, 110.6, 81.1, 65.7, 55.3, 38.7, 36.4, 12.1 , (only 13 of the 14 expected resonances were observed). EI-MS: $m / z 641\left(66,[\mathrm{M}+1]^{+}\right)$, $568(100)$.

Compound 6a. A solution of 5a (370 $\mathrm{mg}, 1.00 \mathrm{mmol})$ and 1,4-dimethoxybenzene $(152 \mathrm{mg}$, $1.10 \mathrm{mmol})$ in TFA $(5 \mathrm{~mL})$ was stirred and heated at reflux for $6 \mathrm{~h}$. After rotary evaporation the residue was chromatographed $\left(\mathrm{SiO}_{2}, \mathrm{CHCl}_{3}\right)$ to yield 6a (495 mg, $\left.0.90 \mathrm{mmol}, 90 \%\right)$. M.p. 233-234 ${ }^{\circ} \mathrm{C} .{ }^{1} \mathrm{H}$ NMR $\left(400 \mathrm{MHz}, \mathrm{CDCl}_{3}\right)$ : 7.30-7.15 (m, 4H), 6.73 (s, 6H), 5.29 (d, J= 15.6, $2 \mathrm{H}), 4.79(\mathrm{~d}, J=16.0,2 \mathrm{H}), 4.41(\mathrm{~d}, J=16.0,2 \mathrm{H}), 4.30-4.10(\mathrm{~m}, 6 \mathrm{H}), 3.77(\mathrm{~s}, 6 \mathrm{H}), 1.35-1.20$ $(\mathrm{m}, 6 \mathrm{H})$. 
Compound 6c. A solution of 5c (370 mg, $1.00 \mathrm{mmol})$ and 1,4-dimethoxybenzene $(152 \mathrm{mg}$, $1.10 \mathrm{mmol})$ in TFA $(5 \mathrm{~mL})$ was stirred and heated at reflux for $6 \mathrm{~h}$. After rotary evaporation the residue was chromatographed $\left(\mathrm{SiO}_{2}, \mathrm{CHCl}_{3}\right)$ to yield $\mathbf{6 c}(554 \mathrm{mg}, 0.96 \mathrm{mmol}, 96 \%)$. M.p. 249-251 ${ }^{\circ} \mathrm{C}$. TLC $\left(\mathrm{CHCl}_{3} / \mathrm{MeOH}, 100: 1\right) R_{\mathrm{f}} 0.48$. IR $\left(\mathrm{KBr}, \mathrm{cm}^{-1}\right): 2975 \mathrm{w}, 2937 \mathrm{w}, 1755 \mathrm{~s}$, $1720 \mathrm{~s}, 1462 \mathrm{~s}, 1262 \mathrm{~s}, 1079 \mathrm{~m} .{ }^{1} \mathrm{H}$ NMR $\left(400 \mathrm{MHz}, \mathrm{CDCl}_{3}\right): 7.00(\mathrm{~s}, 2 \mathrm{H}), 6.41(\mathrm{~s}, 2 \mathrm{H}), 5.33$ $(\mathrm{d}, J=16.0,2 \mathrm{H}), 4.71(\mathrm{~d}, J=16.0,2 \mathrm{H}), 4.34(\mathrm{~d}, J=16.0,2 \mathrm{H}), 4.26(\mathrm{q}, J=7.2,2 \mathrm{H}), 4.17(\mathrm{q}$, $J=7.2,2 \mathrm{H}), 4.12(\mathrm{~d}, J=16.0,2 \mathrm{H}), 1.35-1.20(\mathrm{~m}, 6 \mathrm{H}) .{ }^{13} \mathrm{C}$ NMR $\left(100 \mathrm{MHz}, \mathrm{CDCl}_{3}\right): 166.0$, 155.9, 150.7, 135.9, 133.7, 130.9, 126.2, 111.8, 80.3, 80.1, 63.1, 62.9, 56.5, 45.0, 37.0, 18.9, 13.8, 13.8. EI-MS: $m / z, 579\left(68,[\mathrm{M}+1]^{+}\right), 506(100)$.

\section{References:}

(1) Huang, S.; Pan, Y.; Zhu, Y.; Wu, A. Org. Lett. 2005, 7, 3797-3799.

(2) Wu, A.; Chakraborty, A.; Witt, D.; Lagona, J.; Damkaci, F.; Ofori, M. A.; Chiles, J. K.; Fettinger, J. C.; Isaacs, L. J. Org. Chem. 2002, 67, 5817-5830.

(3) Burnett, C. A.; Lagona, J.; Wu, A.; Shaw, J. A.; Coady, D.; Fettinger, J. C.; Day, A. I.; Isaacs, L. Tetrahedron 2003, 59, 1961-1970.

(4) Diederich, F.; Jonas, U.; Gramlich, V.; Herrmann, A.; Ringsdorf, H.; Thilgen, C. Helv. Chim. Acta 1993, 76, 2445-2453.

(5) Shahak, I.; Bergmann, E. D. J. Chem. Soc. C 1966, 1005-1009.

(6) Kang, J.; Hilmersson, G.; Santamaría, J.; Rebek, J. J. J. Am. Chem. Soc. 1998, 120, 3650-3656.

(7) Ardecky, R. J.; Kerdesky, F. A. J.; Cava, M. P. J. Org. Chem. 1981, 46, 1483-1485.

(8) Isaacs, L.; Witt, D. Angew. Chem. Int. Ed. 2002, 41, 1905-1907.

(9) Witt, D.; Lagona, J.; Damkaci, F.; Fettinger, J. C.; Isaacs, L. Org. Lett. 2000, 2, 755758.

(10) Isaacs, L.; Fettinger, J. C. Chem. Commun. 1999, 2549-2550. 
$882^{\circ} \cdot \tau$

$\downarrow 乙 \varepsilon \cdot \tau$

0 โโ $\cdot$

$D E \tau \cdot 乙$
$09 \tau \cdot 乙$

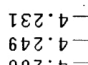

.

$582 \cdot b$

$62 \varepsilon^{\circ}$

$69 \varepsilon^{\circ} \cdot$

$189^{\circ} \cdot$

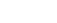

$000^{\circ} 2$

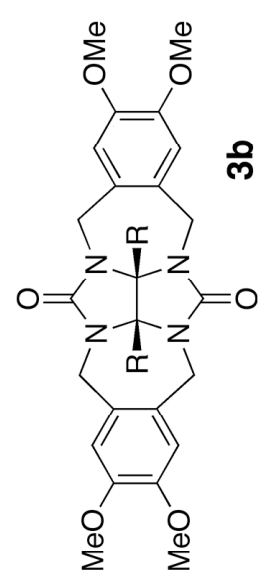

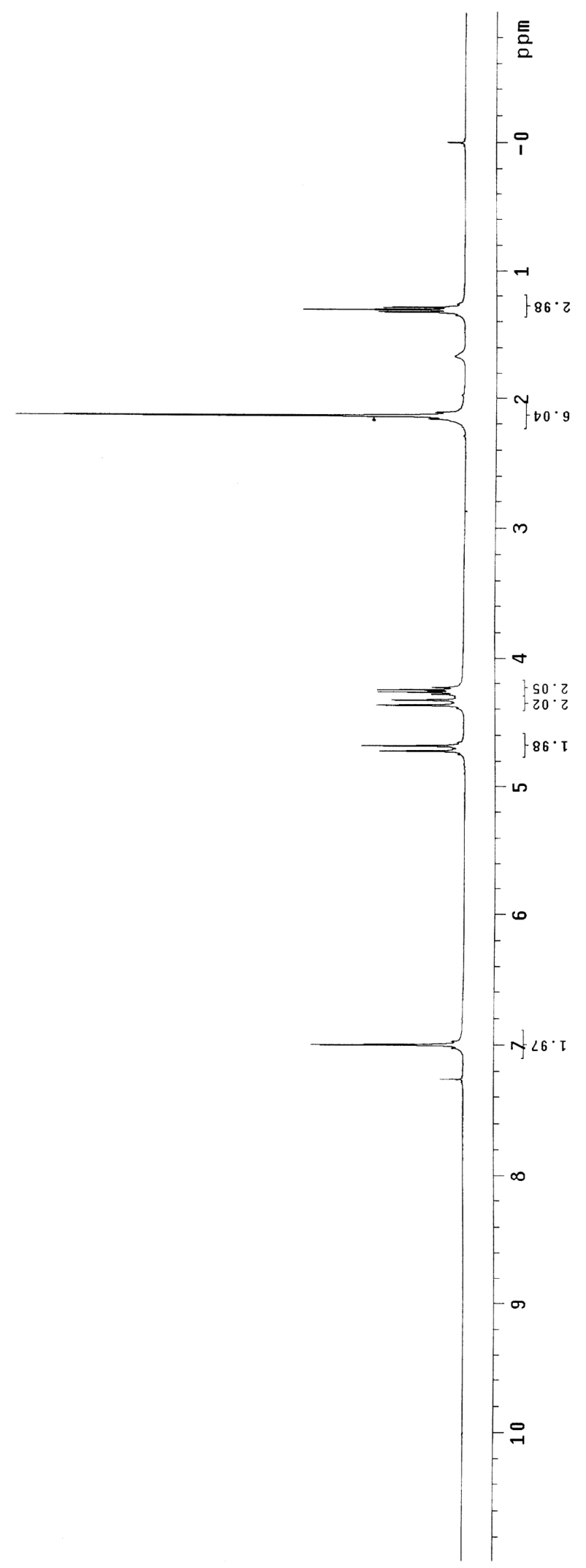



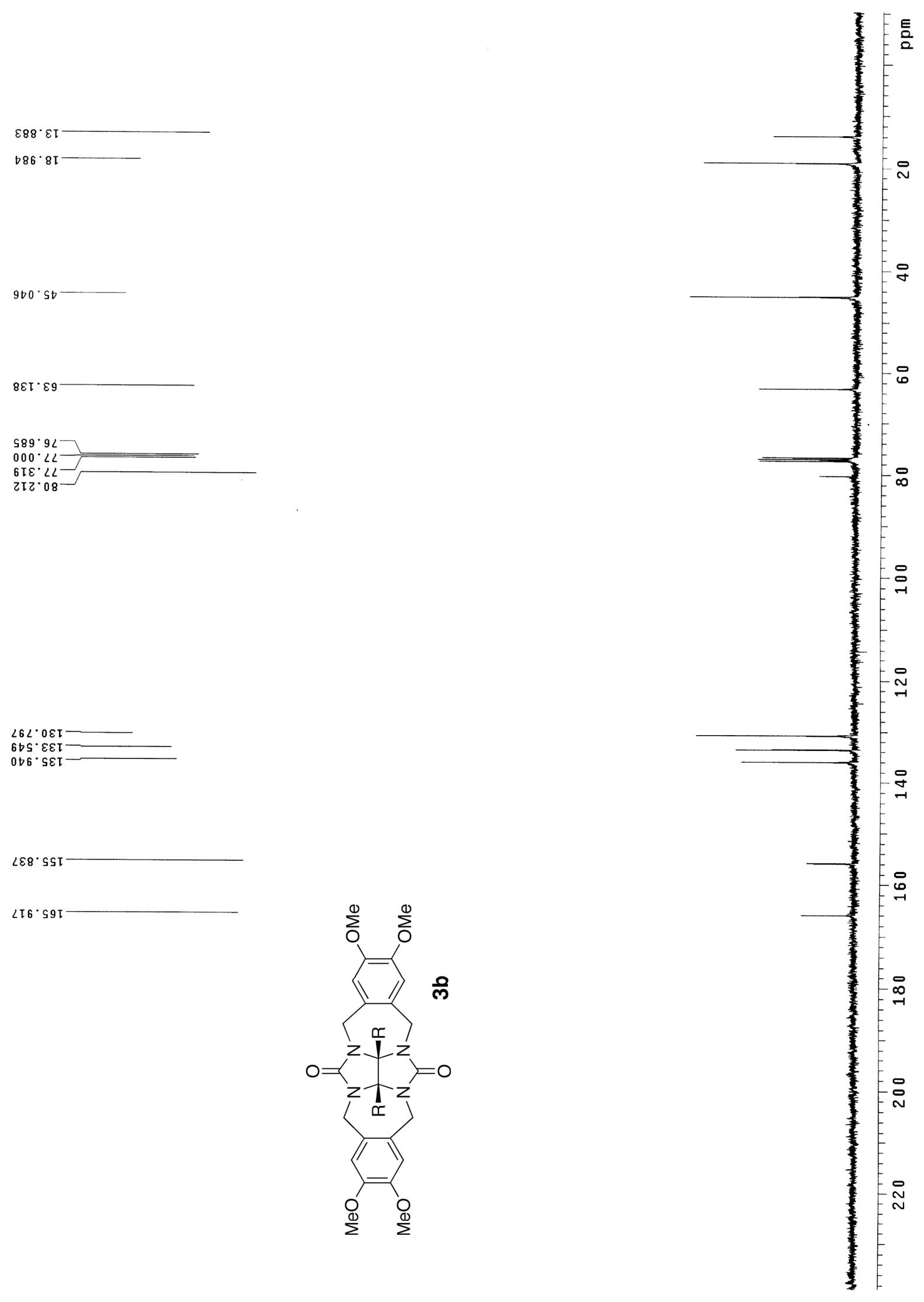

$\angle \varepsilon 8 \cdot S S I$

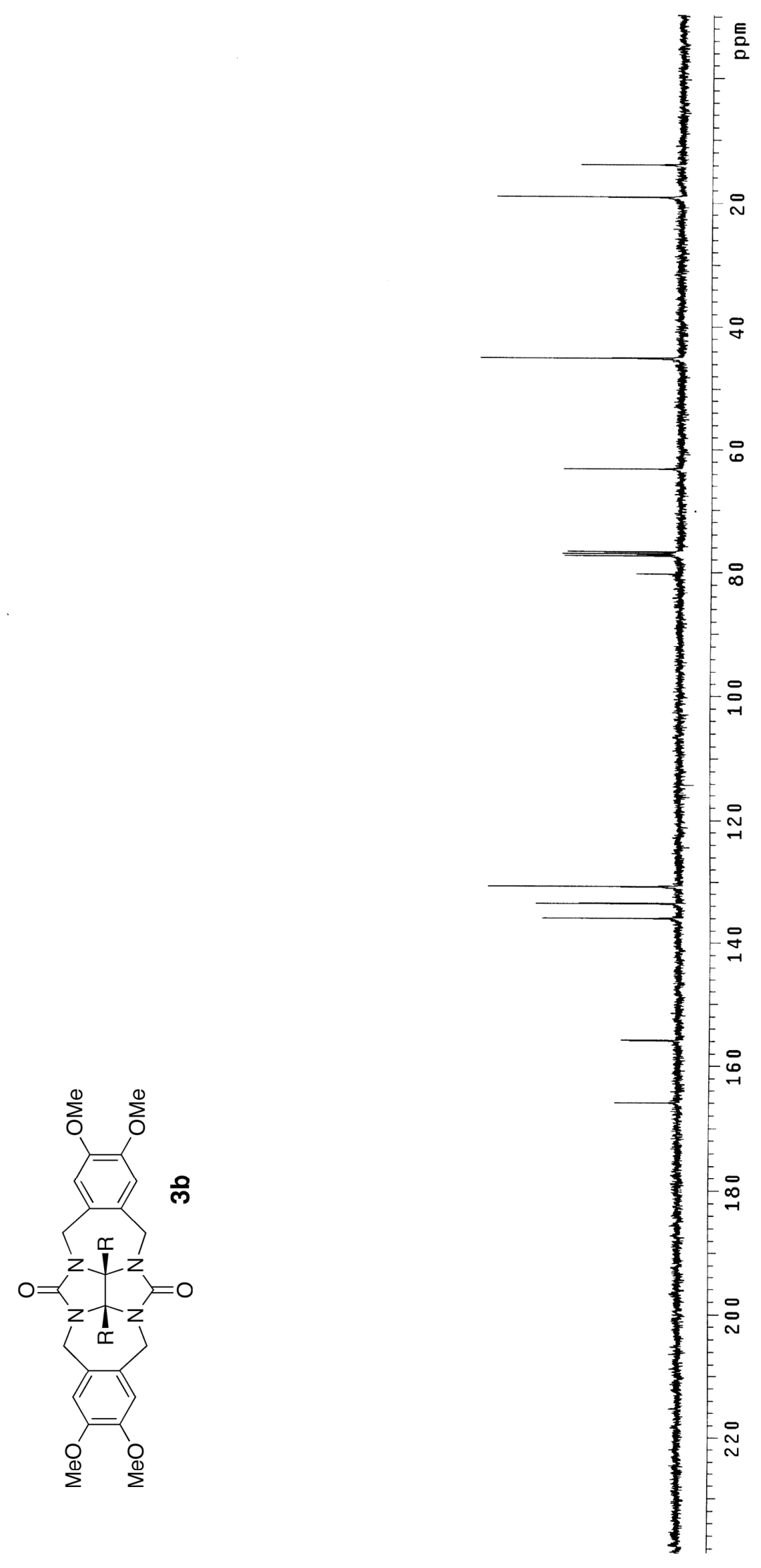




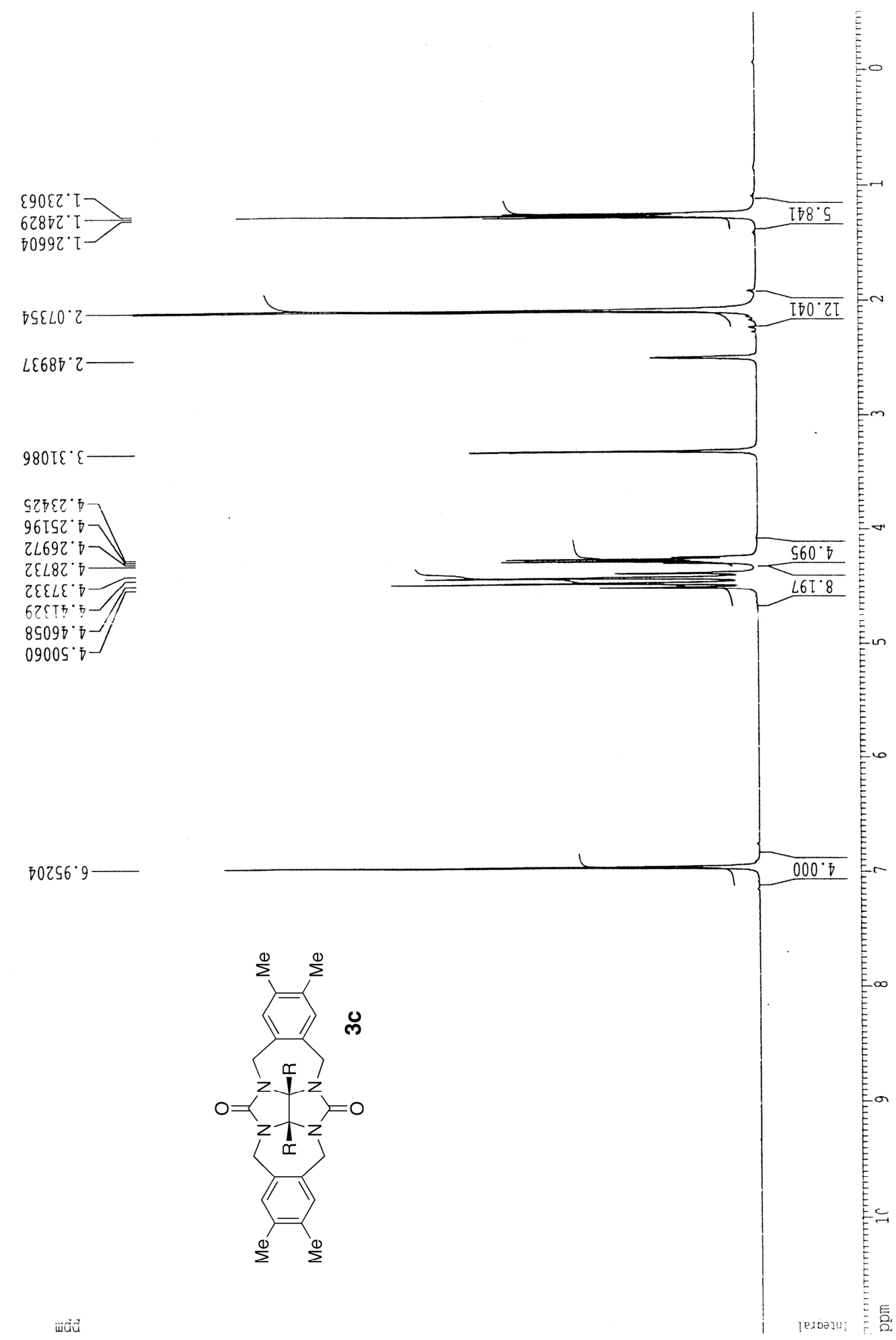




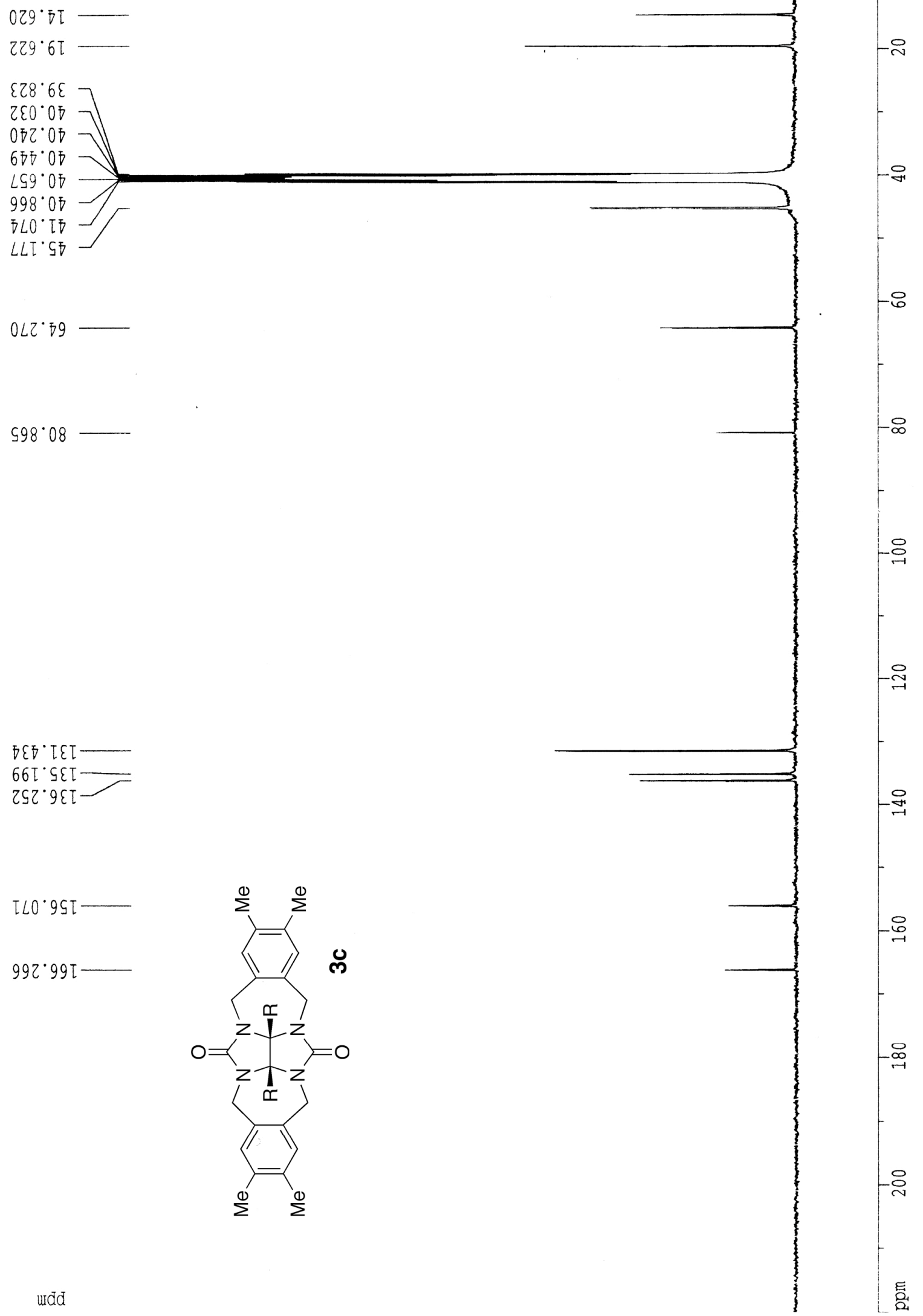


๖ $9 \varepsilon^{\circ} \mathrm{I}$

उ8. 1

$66 \varepsilon^{\circ} \mathrm{I}$

$0 \sqcup \varepsilon^{\circ} b$

$8 S \varepsilon^{\circ} \circ$

$6 \varepsilon^{\circ} \circ$

$580^{\circ} \circ$

I 60.0

$8 \angle 8^{\circ} b$

$068^{\circ} \mathrm{b}$

โI 6.0

$\angle 92 \cdot 2$

IIt.

$5 \angle 6^{\circ} \angle$

$286^{\circ}<$

200.8

$601 \cdot 8$

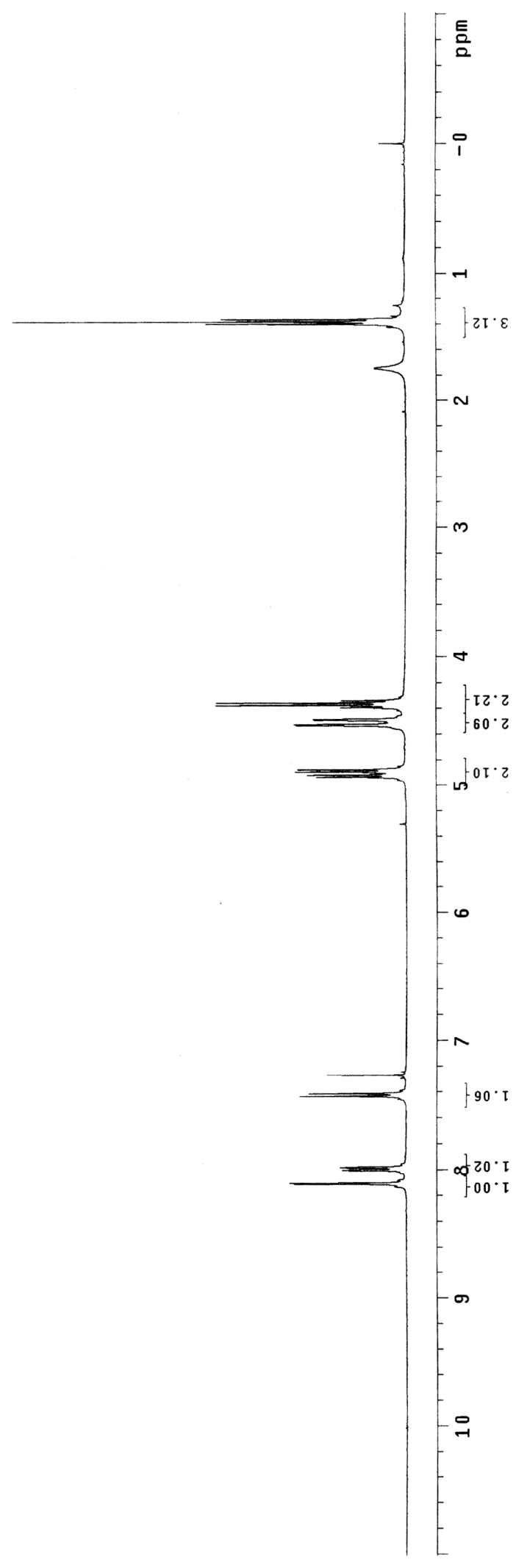



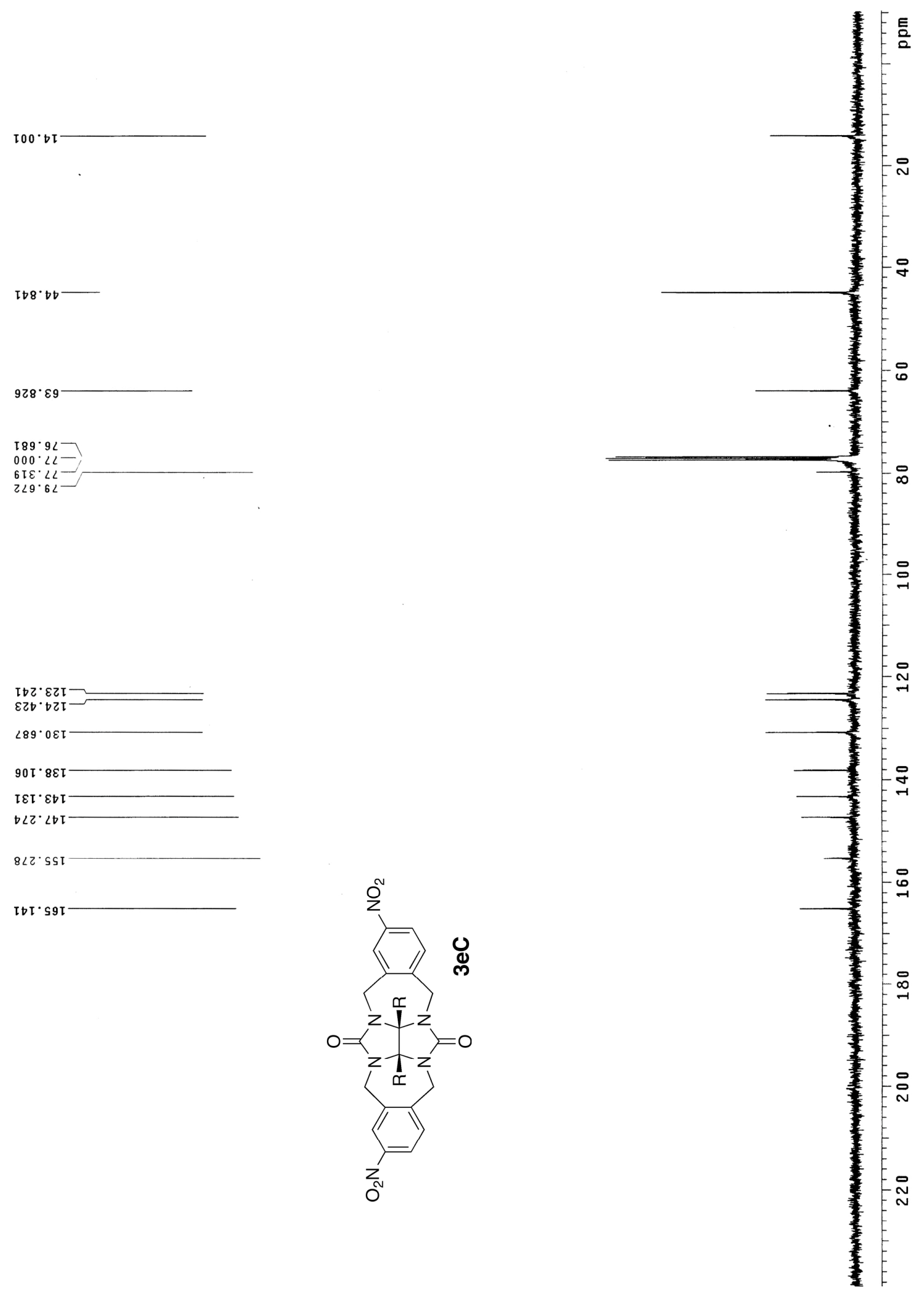
$\varepsilon 9 \varepsilon \cdot \tau-$

$08 \varepsilon^{\circ} \tau$

Esg.

$6 \varepsilon \varepsilon \cdot b$

$9 s \varepsilon^{\circ}$,

$S L E$.

ए

$580^{\circ} \circ$

925,

$088^{\circ} \circ$

$088^{\circ} \circ$

026.5

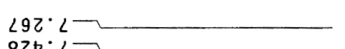

$82 t \cdot 2$

$600 \cdot 2$

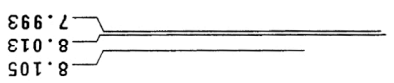

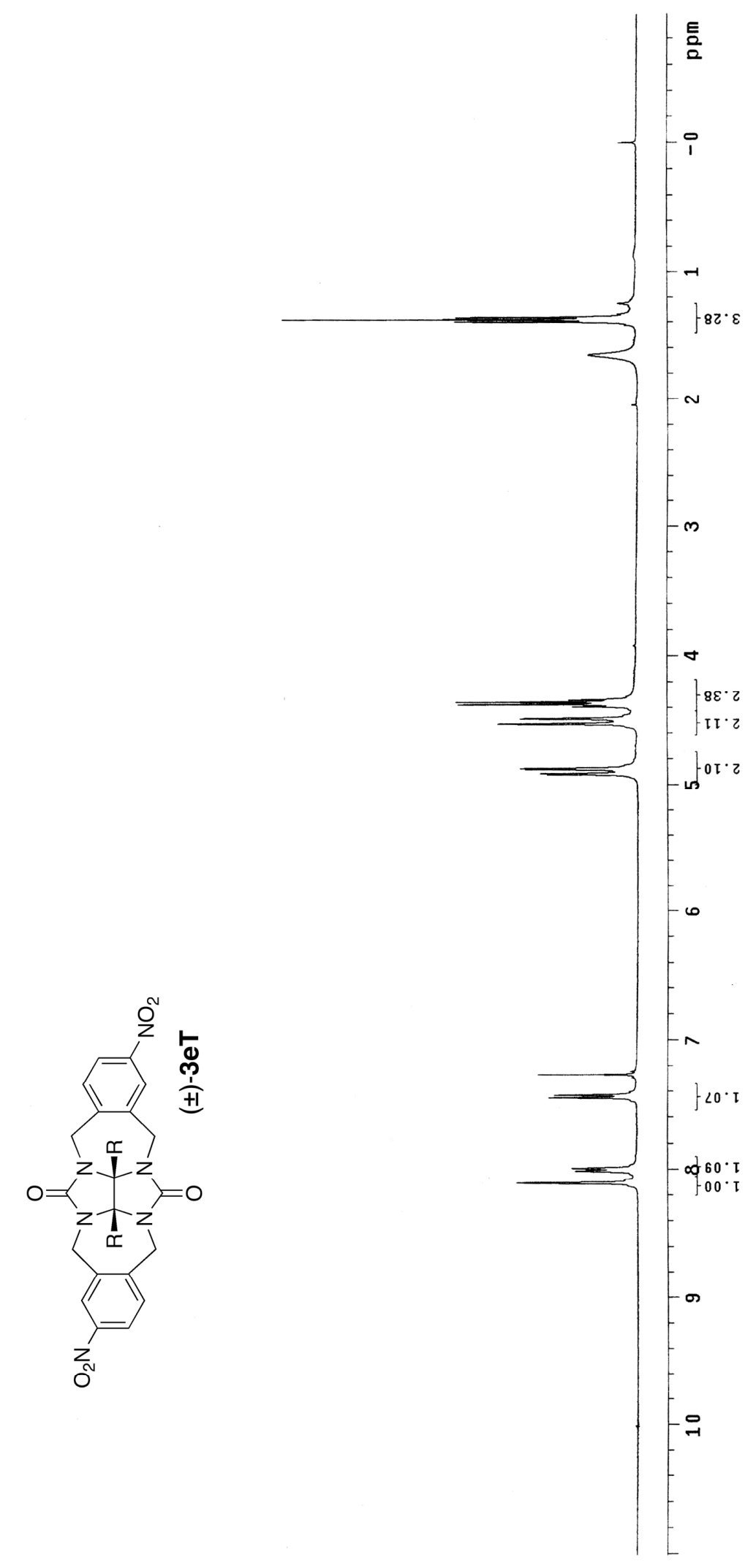




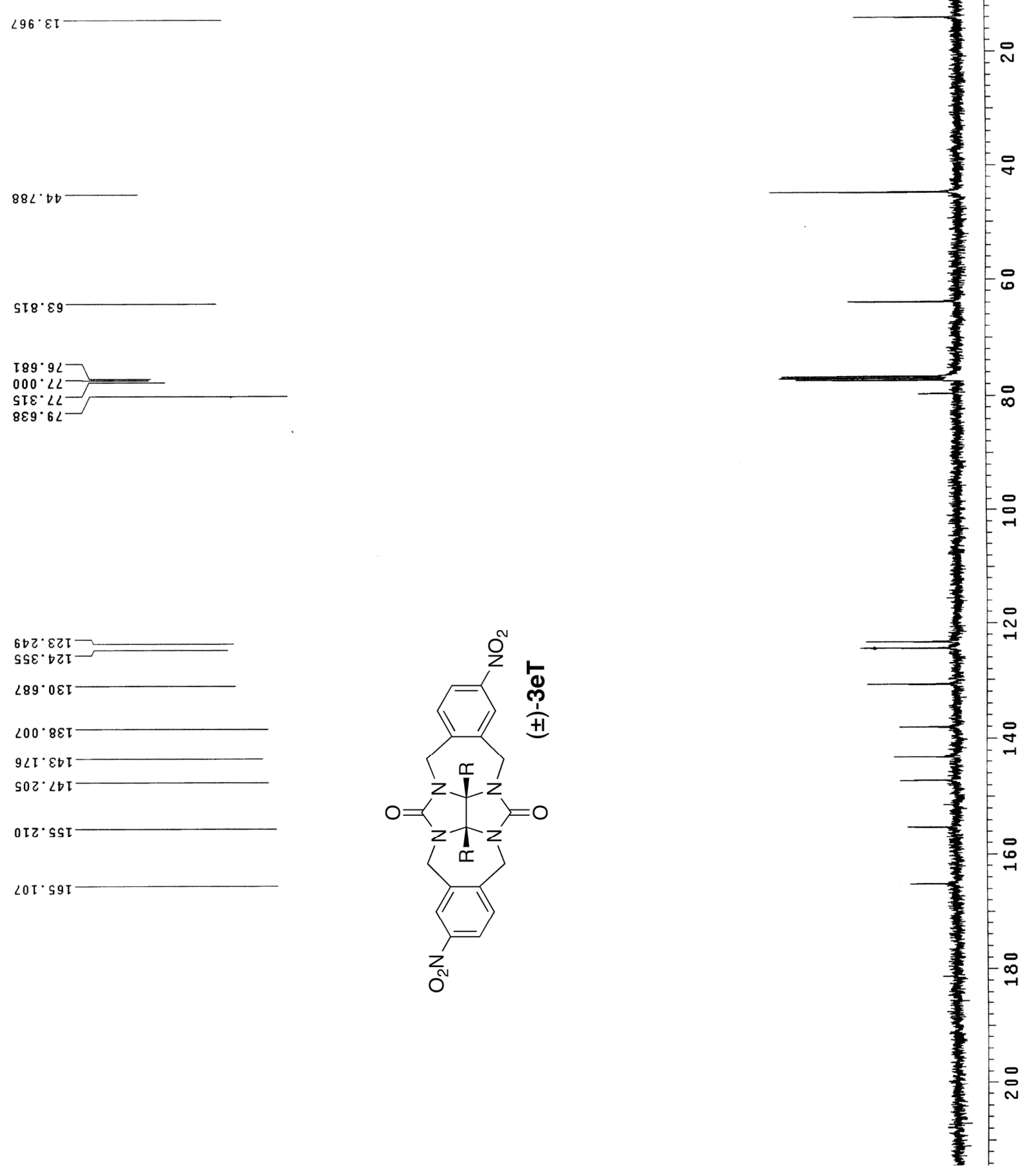


$000.0--$

โ $92^{\circ} \mathrm{T}$

$\angle \measuredangle 2 \cdot \tau-=$

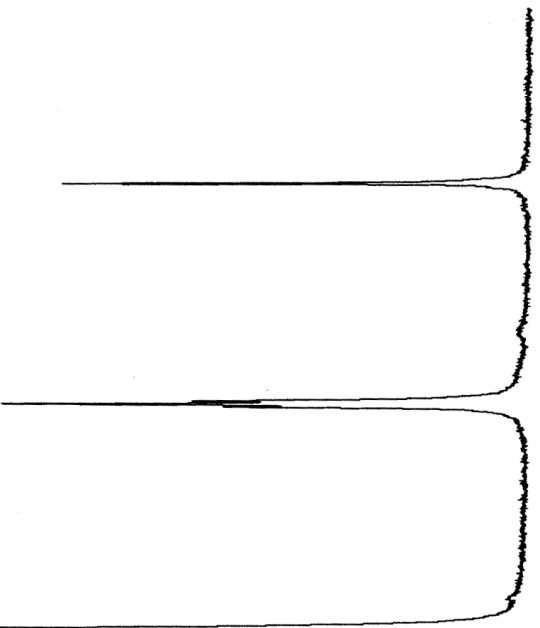

095:2-

$0 \angle 8^{\circ} \varepsilon$

$\rightarrow 68^{\circ} \varepsilon$

$\varepsilon 92^{\circ} \circ$

$6 \angle Z^{\circ} b$

$\checkmark 9 \varepsilon \cdot b$

St 9.0

$\angle 89^{\circ} \circ$

$6 \mathrm{SI} \cdot \mathrm{s}$

$00 z \cdot s$

sहE. $s$

$\angle 80^{\circ} \cdot 2$
$0 I 1 \cdot L$

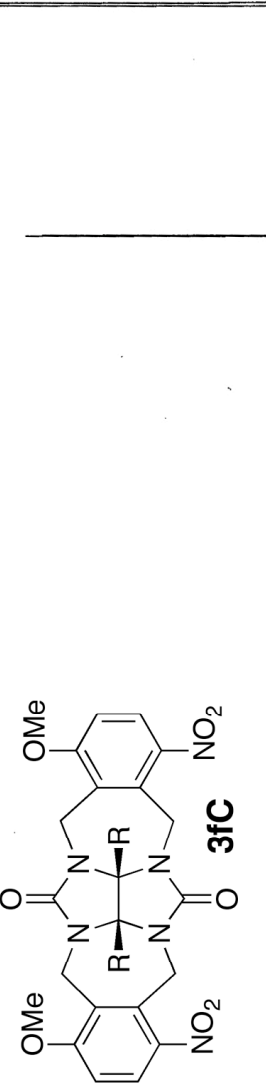

$\varepsilon 6 L^{\circ}$

SI8. L
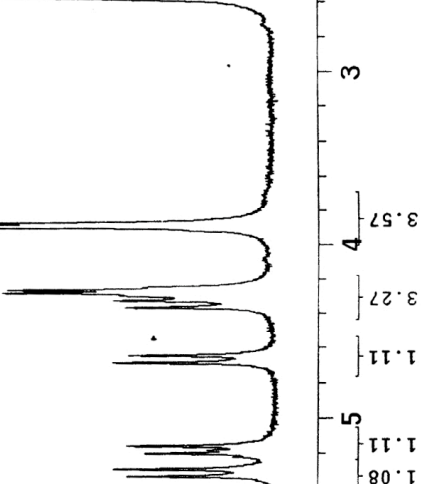

$-80^{\circ} \tau$

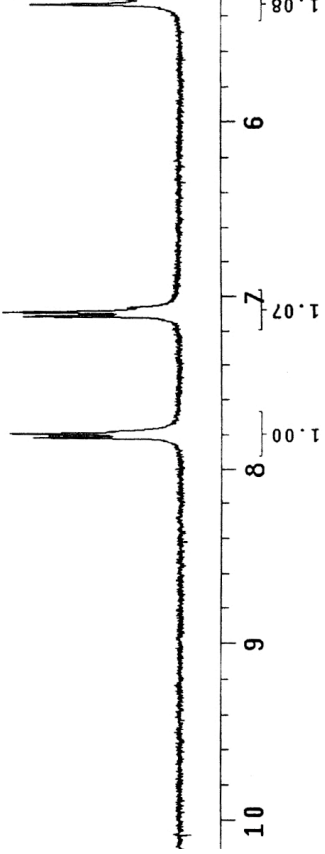


$\angle I I \cdot Z I$

$2 \angle t \cdot 9 \varepsilon$

$908 \cdot 8 \varepsilon$

sze.ss

$8 \mathrm{SL} 99$

$\varepsilon 9 \tau \cdot \tau_{8}$
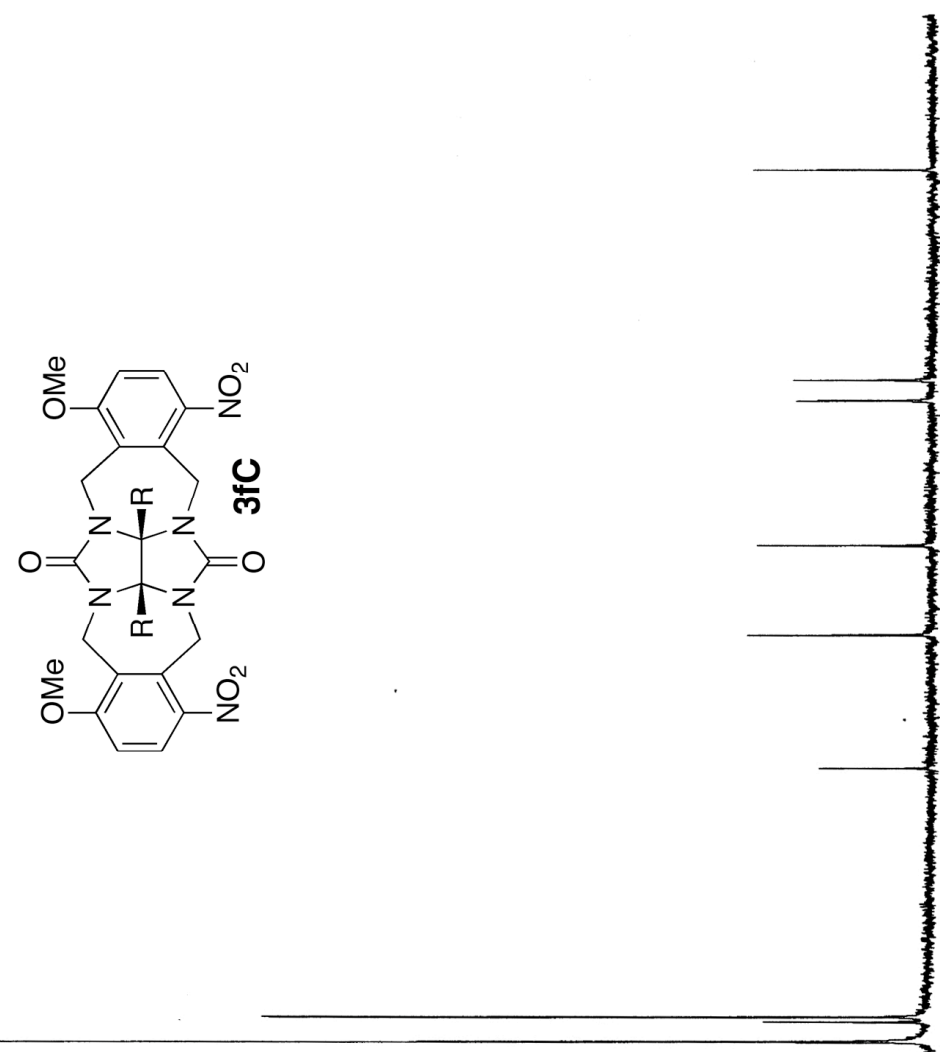

$000.0 \mathrm{II}$

$0 \angle S^{\circ} 0$ II

6ร2.

$9 D t \cdot 8$ II

DI $6.52 I$

$S 8 \varepsilon^{\circ} \angle Z I$

$\varepsilon 己 \iota^{\cdot} \varepsilon \varepsilon \tau$

$0 \angle I \cdot Z D I \longrightarrow$

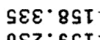

$9 \varepsilon 2 \cdot 6 \mathrm{~s}$

$\angle$ โ8.09โ

$\angle 0 b^{\circ}$. 91

$0 \circ 8^{\circ} 19$

$\angle L 2{ }^{\circ} 291$
$8 \varepsilon 8^{\circ} 591$
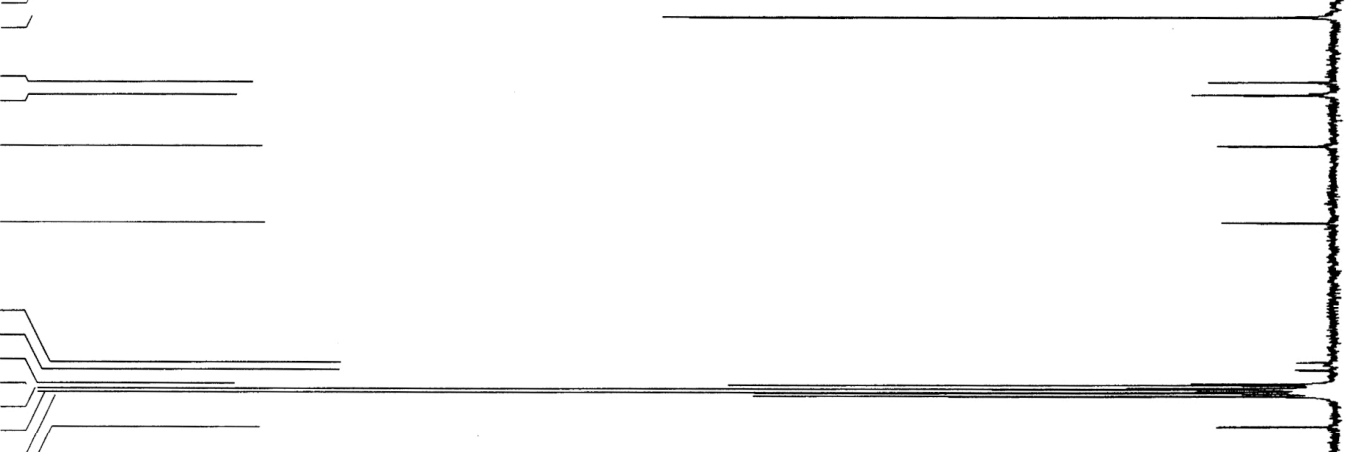

$\stackrel{0}{0}$

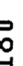

$\rightarrow$

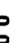

N 
$000^{\circ} 0--$

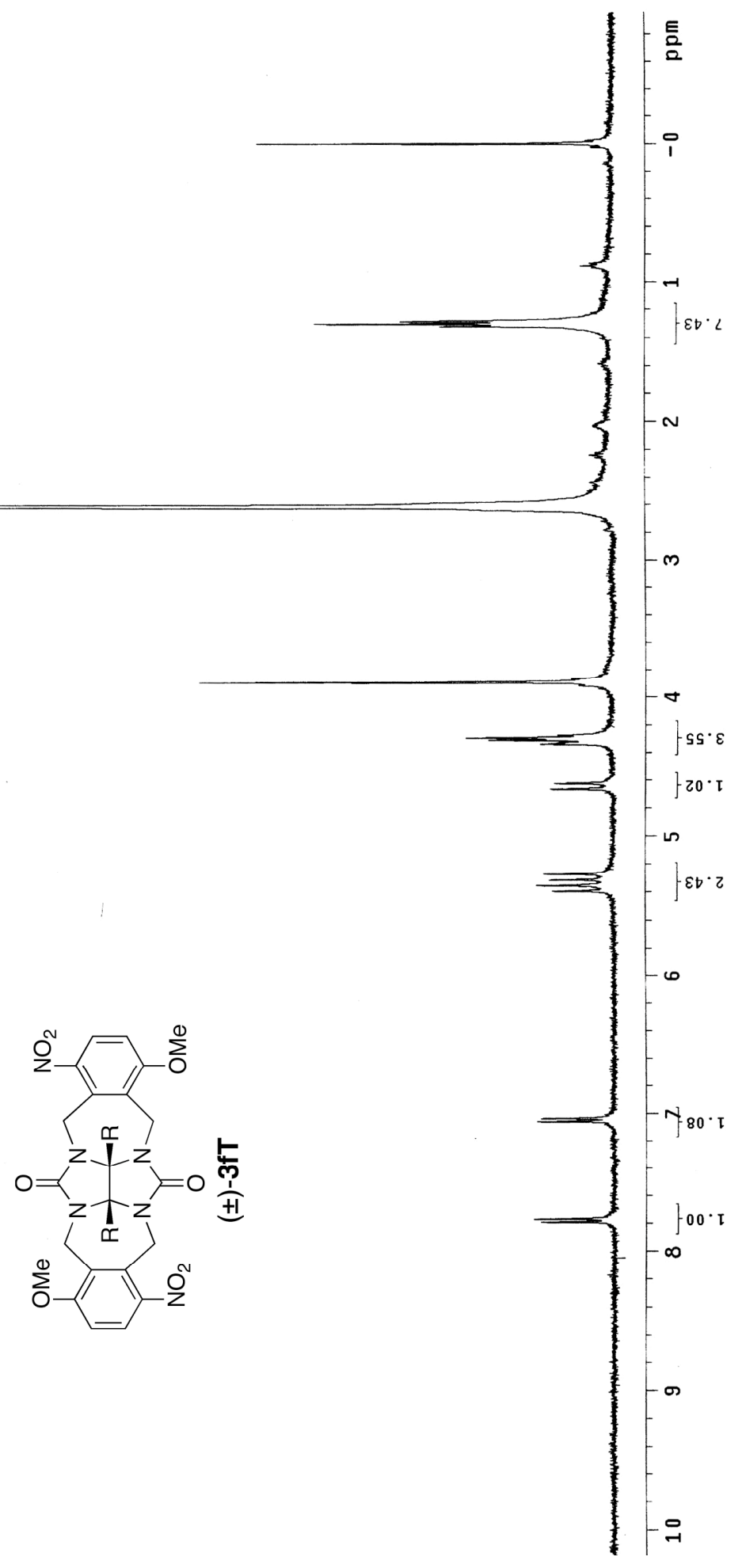

$068^{\circ} \varepsilon-$

$s \angle 2 \cdot b$
$862 \cdot b$

6 I $6 \varepsilon^{\circ}$

$8 \varepsilon \varepsilon^{\circ} b$

ร29.

$z<z \cdot s$

ZSE. $s$

$\checkmark 6 \varepsilon \cdot s$

$582 \cdot \tau=$
$\varepsilon 0 \varepsilon^{\circ} \tau-$
$0 Z \varepsilon^{\circ} \tau-$

$19 \cdot 2-$

$89 L L$
$06 L$

$S 50^{\circ} L$
$850^{\circ} L$ 


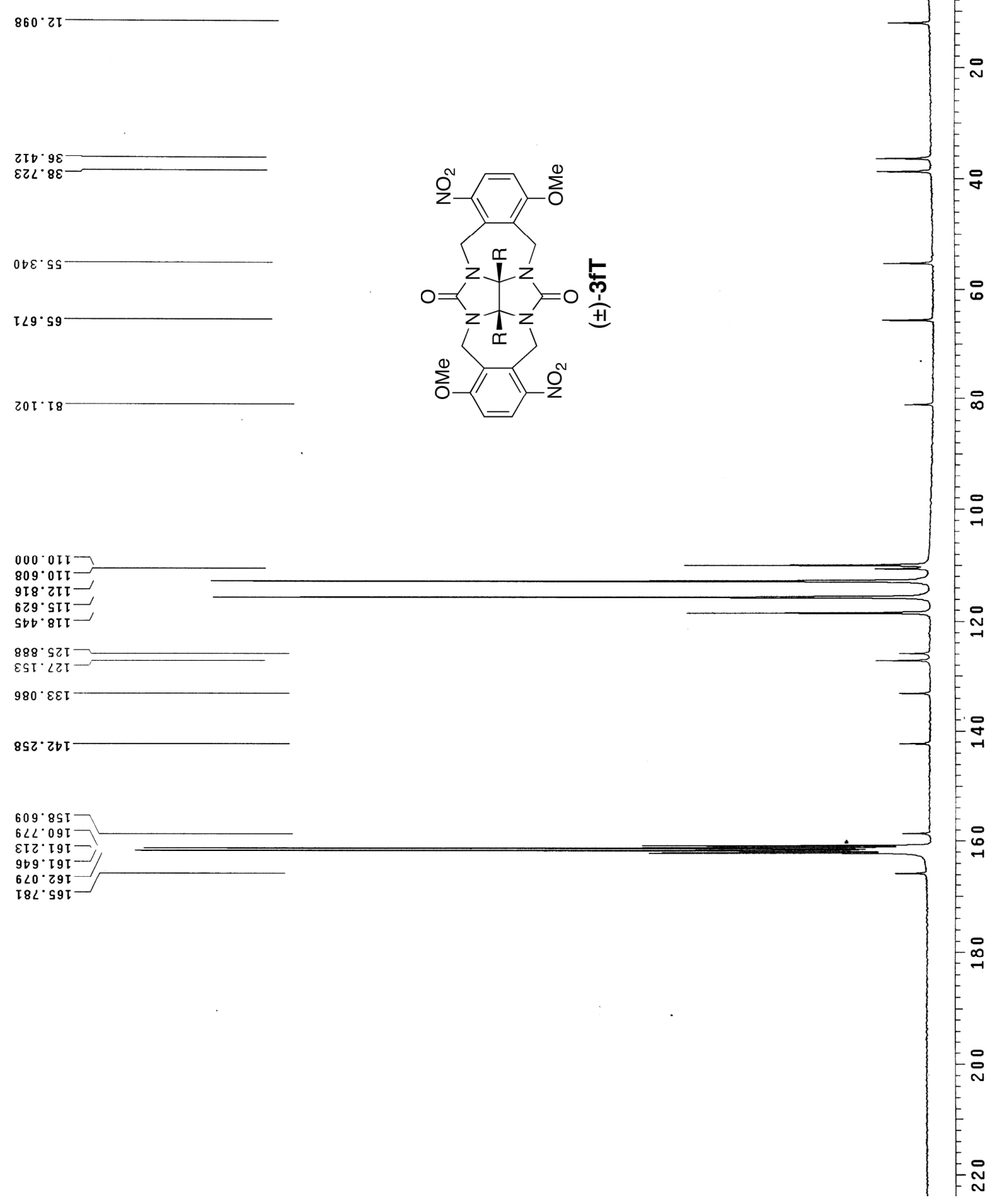



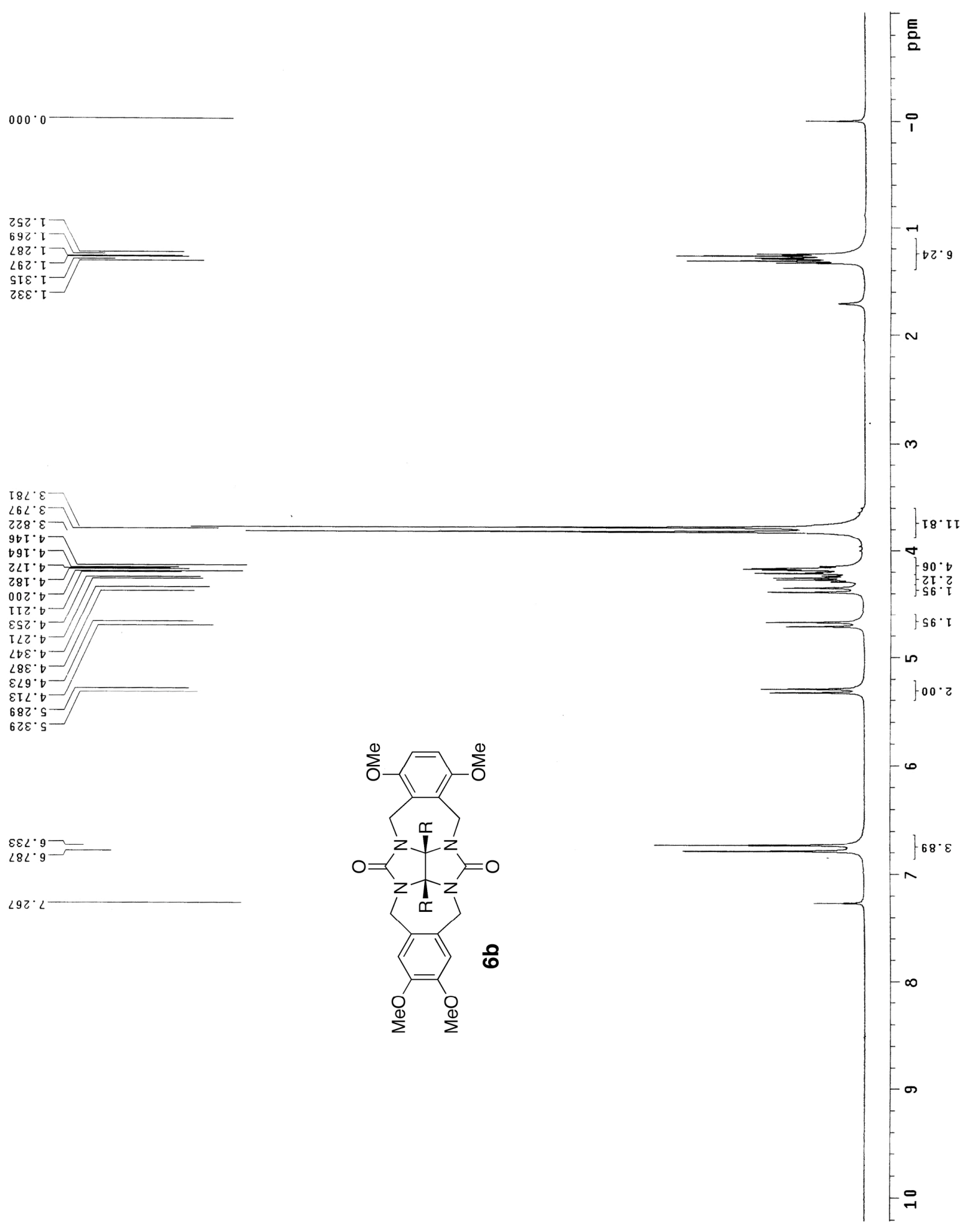

SIE. $T$

โ8L 8

$\angle 6 L \cdot$

$228^{\circ} \varepsilon$

ZLI.

$28 \mathrm{I} \cdot \mathrm{D}$

002.5

IL $Z^{\circ} b^{\circ}$

I $\angle 2{ }^{\circ} D$

$\angle D E \cdot D$

$\angle 8 \varepsilon^{\circ} \circ$

$\varepsilon \angle 9^{\circ} \circ-$

$\varepsilon I L^{\circ} \cdot$

$62 \varepsilon \cdot s$

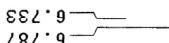

$\angle 9 Z^{\circ} \angle$ 


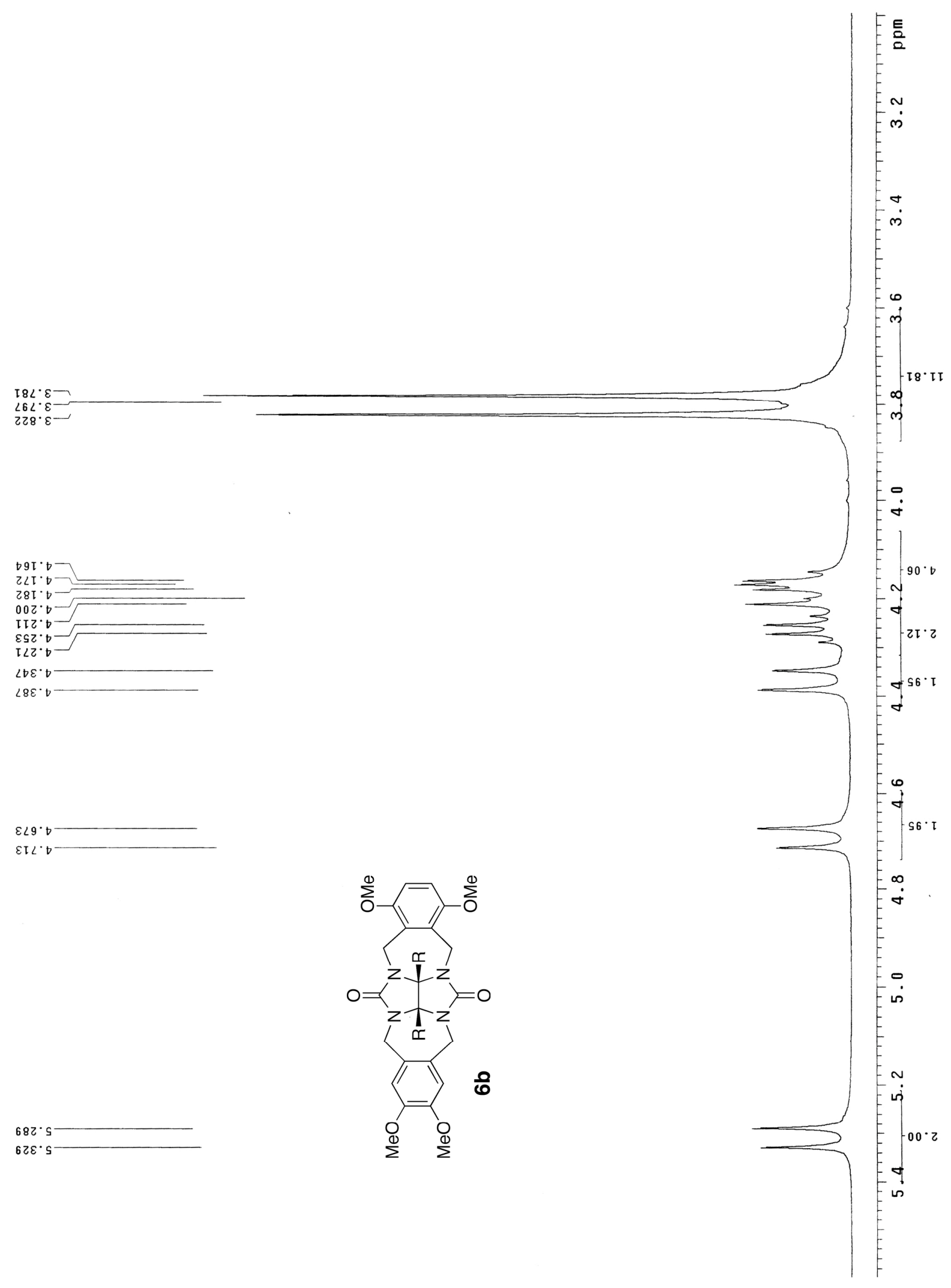


$0 \mathrm{I} 6^{\circ} \varepsilon \mathrm{I}$

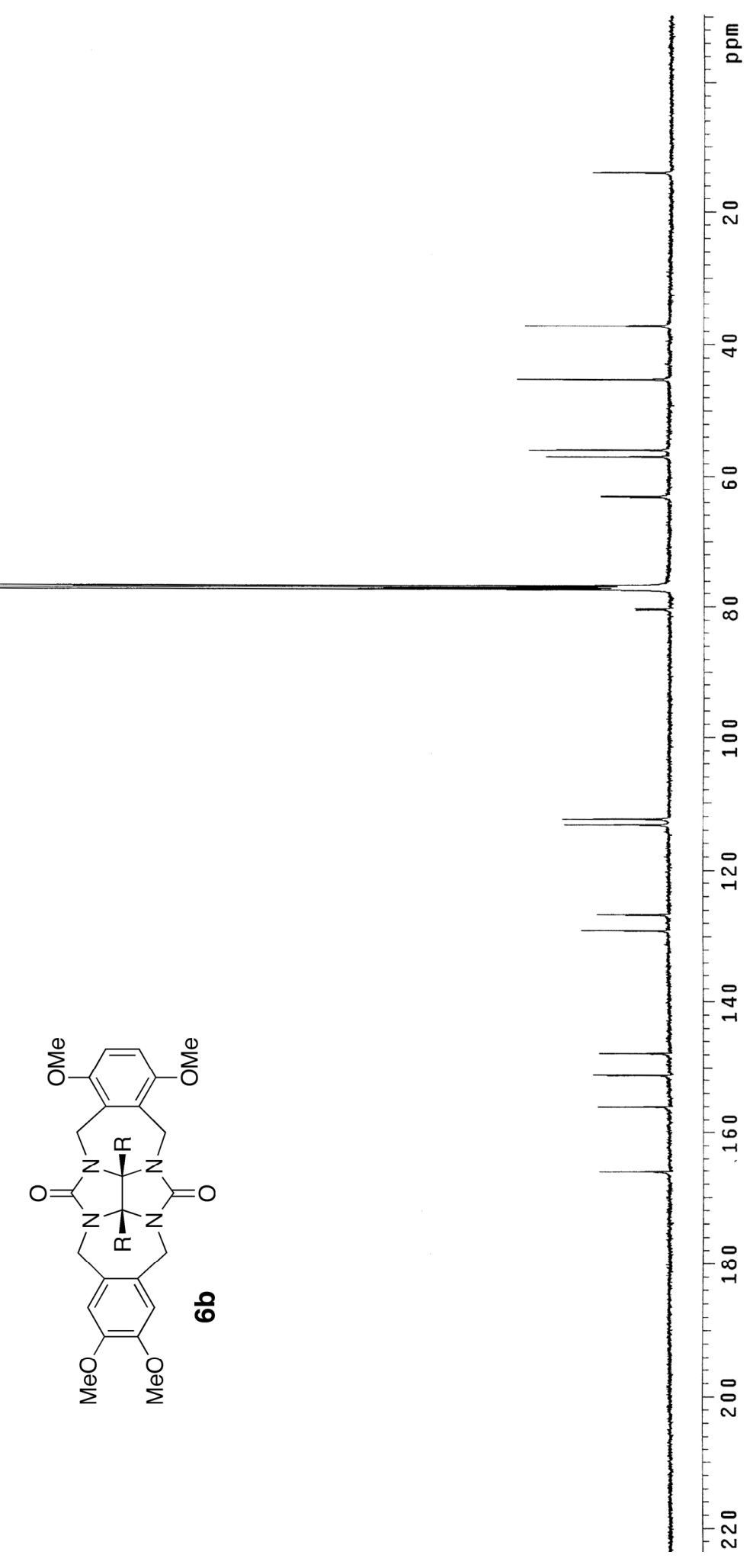

$50 z \cdot<\varepsilon$

$9 \angle I \cdot S B$

$826.5 s^{-}$

996.95

$8<0^{\circ} \varepsilon 9$

SDZ $\varepsilon 9$

$589 \cdot 9 L$

$000^{\circ} \angle L$

6 I $\varepsilon^{\circ}<2$

$202 \cdot 08$

000.08

$6 S E \cdot 2 I T-$

โ $乙 \cdot \varepsilon \tau \tau$

โ $89^{\circ} 9 Z \tau$

$89 \angle{ }^{\circ} \angle b T$

$S 8 I^{\circ} \tau S T$

sE0.9s

$880 \cdot 991$

g

$\underset{-1}{0}$

$\underset{\infty}{\infty}$

只

요 
$002 \cdot 1$
$852 \cdot 1$

$\angle \angle 2 \cdot \tau$

$9 \tau \varepsilon \cdot \tau$

$6 \varepsilon 0 \cdot z$

$20 L \varepsilon^{\circ}$

$2 \varepsilon \tau^{\circ} \cdot$

$99 I^{\circ} \cdot b$

$6 b 2 \cdot b$

$992^{\circ} b$

七टा.

s89.

589.

$\angle 0 \varepsilon^{\circ} s$

$600 \cdot 9$

$100 \%$

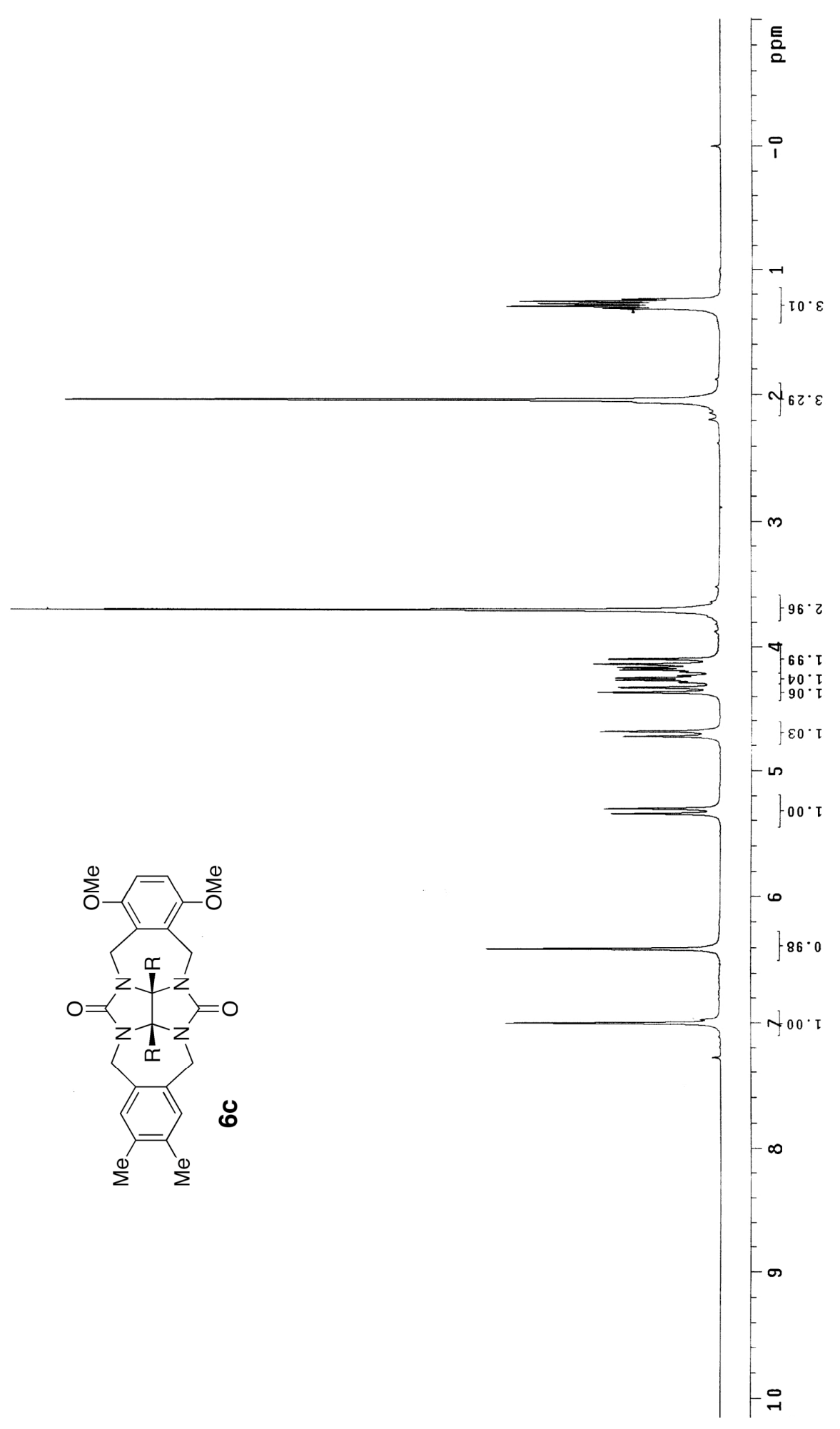


$860.6-$

$\angle E I \cdot D-$

$99 \tau^{\circ} \mathrm{b}$

เ $102 \cdot b$

IEz $b$

$602 . b$

$\downarrow \tau \varepsilon \cdot b$

$s 9 \varepsilon^{\circ}-$

$s 89^{\circ} \mathrm{b}$

$s 2 L \cdot b$

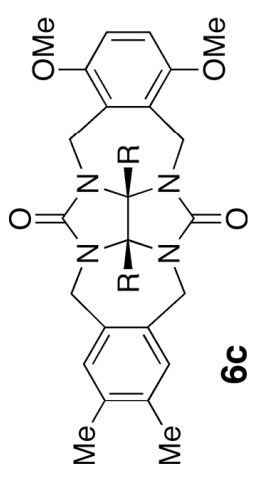

$\angle 0 \varepsilon^{\circ} \mathrm{s}$

$\angle D E \cdot s-$

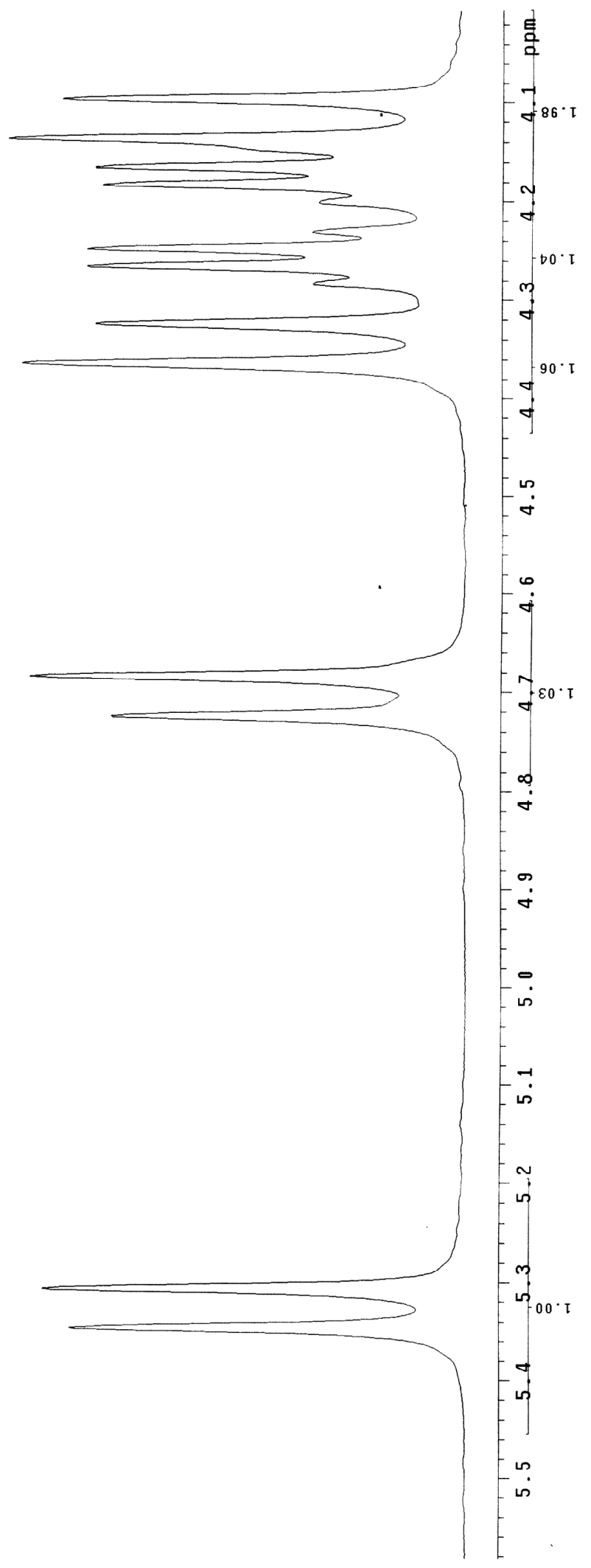


$008^{\circ} \varepsilon I$

โ $8 . \varepsilon \tau$

$806 \cdot 8 I$

$0 \angle 6 \cdot 9 \varepsilon^{-}$

$986^{\circ} \cdot t b$

$8 \mathrm{DS} \cdot 9 \mathrm{~s}$

$\angle \varepsilon 6 \cdot 29$
$9 \tau \cdot \cdot \varepsilon 9$

โ $89.9 L$

$000^{\circ} \mathrm{LL}$

LI 2

$\angle 90.08$
S92.08

วโ8・โโโ

$0 D Z \cdot 9 Z I$

$\varepsilon \angle 8^{\circ} 0 \varepsilon \tau$

$\varepsilon 69^{\circ} \varepsilon \varepsilon \mathrm{I}$

$868^{\circ} \mathrm{SEI}$

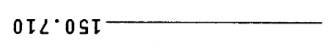

$\angle 98^{\circ}$ SSI

$000 \cdot 99$ I
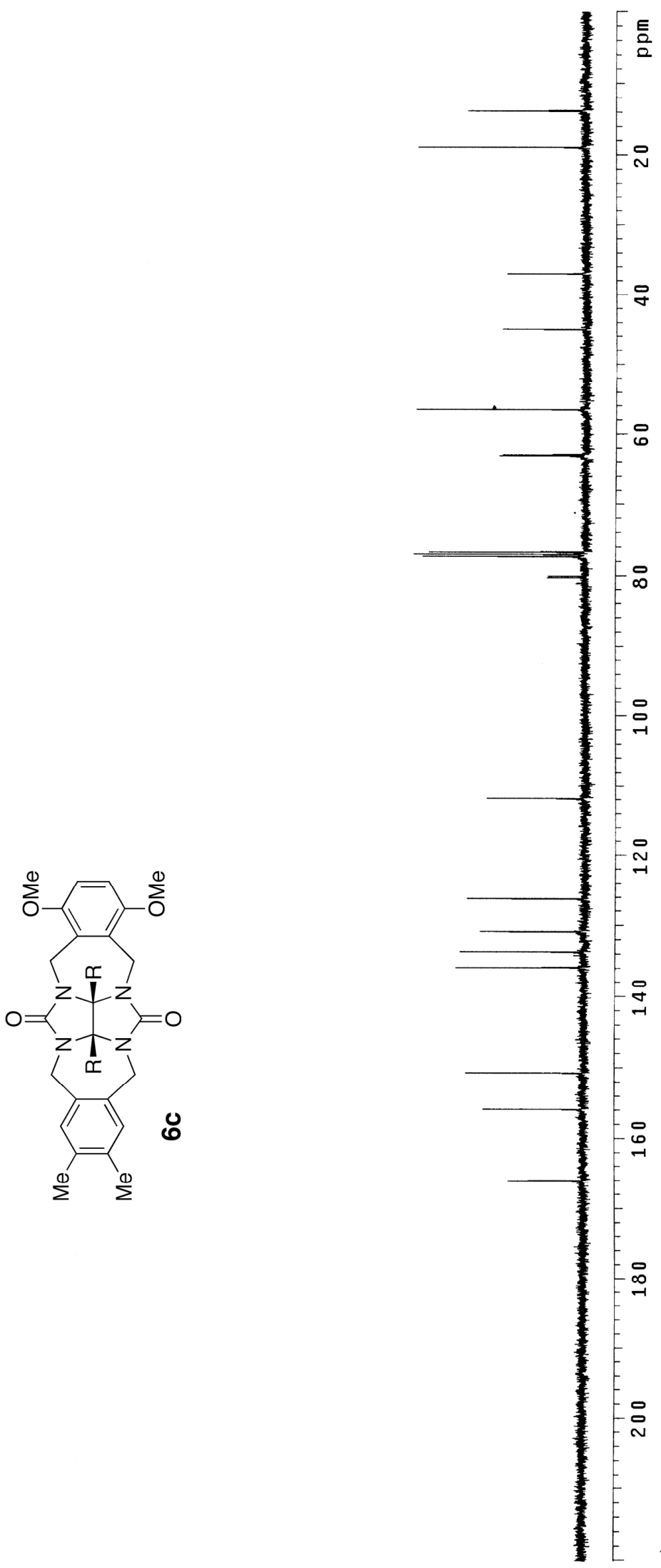